\title{
Resveratrol ameliorates brain injury via the TGF- $\beta$-mediated ERK signaling pathway in a rat model of cerebral hemorrhage
}

\author{
RIGUANG ZHAO ${ }^{1}$, KUN ZHAO $^{1}$, HONGJUN SU ${ }^{1}$, PENG ZHANG $^{2}$ and NA ZHAO ${ }^{1}$ \\ ${ }^{1}$ Baodi Clinical College of Tianjin Medical University, Tianjin Baodi Hospital, Tianjin 301800; \\ ${ }^{2}$ College of Basic Medicine, Shanghai Jiao Tong University, Shanghai 240001, P.R. China
}

Received February 8, 2018; Accepted August 2, 2018

DOI: $10.3892 /$ etm.2019.7939

\begin{abstract}
Brain injury is the most common intracranial injury in human cerebrovascular disease, which may lead to ischemic stroke. Resveratrol induces ameliorative effects in the treatment of certain human diseases by regulating different signaling pathways. The present study assessed the therapeutic effects of resveratrol and its potential mechanism of action in the neurons from rats with ischemia/reperfusion-induced cerebral hemorrhage. The rat model of cerebral hemorrhage was established and reverse transcription-quantitative polymerase chain reaction, western blotting, immunohistochemistry and terminal deoxynucleotidyl-transferase-mediated dUTP nick end labeling assays were subsequently performed to assess the therapeutic effects of resveratrol. The results demonstrated that treatment with resveratrol (10 mg/kg/day) decreased cerebral water content, hippocampal cell apoptosis and cerebral infarct volume compared with the PBS-treated group. Resveratrol treatment also increased neuronal cell viability, improved neurological function and blood brain barrier disruption compared with the PBS group following 21 days of treatment. The administration of resveratrol was demonstrated to decrease the levels of certain inflammatory factors, including ionized calcium binding adaptor molecule 1 and myeloperoxidase, in rats with cerebral hemorrhage. The results revealed that treatment with resveratrol regulated neuronal apoptosis by downregulating the transforming growth factor- $\beta$ (TGF- $\beta$ )-mediated extracellular signal-regulated kinase (ERK) signaling pathway. In conclusion, these results indicate that resveratrol decreases ischemia/reperfusioninduced neuronal apoptosis by downregulating the TGF- $\beta$-mediated ERK pathway in a rat model of cerebral
\end{abstract}

Correspondence to: Professor Na Zhao, Baodi Clinical College of Tianjin Medical University, Tianjin Baodi Hospital, 8 Guangchuan Road, Baodi, Tianjin 301800, P.R. China

E-mail: nazhao_z@126.com

Key words: cerebral hemorrhage, resveratrol, apoptosis, transforming growth factor- $\beta$, extracellular signal-regulated kinase hemorrhage and may serve as a potential agent for the treatment of cerebral hemorrhage.

\section{Introduction}

Cerebral hemorrhage is a type of human cardiovascular system disease, which serves an important adjunct role in primary brain injury and often leads to vasospasm (1). A previous systematic review and meta-analysis has indicated that intracranial hemorrhage increases the risk of cerebral microbleeds in patients with acute stroke (2). Cerebrovascular injury is frequently caused by ischemia/reperfusion, which may lead to cerebral infarct and neuronal apoptosis (3). Additionally, the inhibition of neuronal apoptosis is beneficial for the recovery of cognitive disorders in cerebral hemorrhage induced by ischemia/reperfusion (4). Therefore, understanding the apoptosis of neurons is essential for the treatment of cerebral hemorrhage.

Resveratrol is a multifunctional biological polyphenol that serves therapeutic effects in certain types of human disease (5). A previous study has indicated that the neuroprotective effects of resveratrol in ischemic injury are mediated by the improvement of brain energy metabolism and the alleviation of oxidative stress in rats (6). Zou et al (7) have revealed that the continuous infusion of resveratrol over a 4-week period protected neurons from cannulae implantation injury. In addition, pre-treatment with resveratrol attenuated traumatic brain injury in rats by suppressing NACHT, LRR and PYD domains-containing protein 3 inflammasome activation via sirtuin 1 (7). Furthermore, resveratrol also ameliorated hypoxia/ischemia-induced behavioral deficits and brain injury in the neonatal rat brain (8). A previous study has also demonstrated that the decreased expression of the transforming growth factor- $\beta$ (TGF- $\beta$ ) gene superfamily is associated with adult neurogenic regions following brain injury (9). However, associations between resveratrol and TGF- $\beta$ have not yet been reported in neurons.

The present study assessed the therapeutic role of resveratrol in a rat model of cerebral hemorrhage. Cerebral infarct volume, hippocampal cell apoptosis and neuron viability were analyzed following a 21-day experimental period. Notably, the present study assessed the association between resveratrol and the TGF- $\beta$-mediated extracellular signal-regulated kinase (ERK) signaling pathway in neurons isolated from a rat model of cerebral hemorrhage. 


\section{Materials and methods}

Rat model of cerebral hemorrhage. A total of 16 male Sprague-Dawley rats (weight, 300-330 g; age, 8 weeks) were purchased from Harbin Veterinary Research Institute (Harbin, China). All rats were housed at a temperature of $23 \pm 1^{\circ} \mathrm{C}$ and humidity of $50 \pm 5 \%$, with a 12 -h light/dark cycle and free access to food and water. The rat model of cerebral hemorrhage was established using the modified ischemia/reperfusion method as previously described (10). Rats received right middle cerebral artery occlusion for $90 \mathrm{~min}$ and reperfusion by withdrawal of the filament at $37^{\circ} \mathrm{C}$ Immediately, rats with ischemia/reperfusion-induced cerebrovascular injury were randomly divided into two groups (each, $n=8$ ) and were administered an intravenous injection of resveratrol (10 mg/kg/day; Sigma-Aldrich; Merck KGaA, Darmstadt, Germany) or the same volume of PBS (serving as the control) as described previously (11). The treatments were continued for 20 days. Rats were sacrificed using cervical dislocation following intraperitoneal pentobarbital injection (35 mg/kg body weight) on day 21 for further analysis.

Behavioral tests. Behavioral function (locomotor activity) was examined in experimental rats using open-field tests on day 21. These were used to analyze the efficacy of resveratrol on ischemia/reperfusion injury and were performed as previously described (12).

Cell culture. Rats were sacrificed via cervical dislocation. Neuronal cells were isolated from rats with cerebral hemorrhage prior to treatment as described previously (13). Cells were cultured in RPMI 1640 medium (Thermo Fisher Scientific, Inc., Waltham, MA, USA) supplemented with $10 \%$ heat-inactivated fetal bovine serum (FBS; Thermo Fisher Scientific, Inc.) for $24 \mathrm{~h}$ at $37^{\circ} \mathrm{C}$. Cells were then treated with a TGF- $\beta$ inhibitor (TGF- $\beta$ IR; 2 mg/ml; Sigma-Aldrich; Merck $\mathrm{KGaA}$ ) or TGF- $\beta$ and/or resveratrol ( $2 \mathrm{mg} / \mathrm{ml}$; Sigma-Aldrich; Merck $\mathrm{KGaA}$ ) for $24 \mathrm{~h}$ at $37^{\circ} \mathrm{C}$.

Analysis of cerebral water content (CWC). On day 21, CWC was evaluated in the resveratrol and PBS group as described in a previous study (14). Rat brains were isolated as detailed previously (15). Brains were dried in an electric oven at $100^{\circ} \mathrm{C}$ for $24 \mathrm{~h}$ to analyze $\mathrm{CWC}$ in the rat model of intracerebral hemorrhage. The CWC was calculated using the following formula: (wet weight-dry weight/wet weight) x100 (\%).

Quantitative analysis of blood-brain barrier (BBB) permeability. BBB leakage was examined as previously described (16). Briefly, experimental rats received $100 \mu 1$ 5\% Evan's blue with resveratrol or PBS administered intravenously 10 days following ischemia/reperfusion-induced injury. Following the injection of Evan's blue (for $2 \mathrm{~h}$ ), cardiac perfusion was performed under deep anesthesia with $200 \mathrm{ml}$ of saline to clear the cerebral circulation of Evan's blue. Brains were subsequently isolated and sliced. The two hemispheres were homogenized in $750 \mu \mathrm{l}$ of N,N-dimethylformamide (Bio-Rad Laboratories, Inc., Hercules, CA, USA). Quantitative analysis of blood-brain barrier permeability was performed using Evan's blue and absorbance was measured at $689 \mathrm{~nm}$ using a microplate reader.
Cell viability assay. Neuron cell viability was determined using an MTT assay kit (cat. no. M6494; Thermo Fisher Scientific, Inc.), according to the manufacturer's protocol. Neuronal cells $\left(1 \times 10^{3}\right.$ cells/well) were seeded in 96-well plates and cultured in RPMI 1640 medium supplemented with $10 \% \mathrm{FBS}$ at $37^{\circ} \mathrm{C}$ for $22 \mathrm{~h}$. Following incubation, $15 \mu \mathrm{l}$ MTT reagent was added to each well and incubated for a further $4 \mathrm{~h}$. Following incubation, DMSO $(150 \mu \mathrm{l})$ was added to each well to dissolve the purple formazan. Cell viability was determined by measuring the absorbance was measured at a wavelength of $570 \mathrm{~nm}$ using a microplate reader.

Reverse transcription-quantitative polymerase chain reaction (RT-qPCR) analysis. Total RNA was extracted from neuronal cells using an RNAeasy Mini kit (Qiagen Sciences, Inc., Gaithersburg, MD, USA) according to the manufacturer's protocol. RNA was reverse transcribed into cDNA at $42^{\circ} \mathrm{C}$ for $2 \mathrm{~h}$ using the High Capacity cDNA Reverse Transcription kit (Thermo Fisher Scientific, Inc.). PCR amplification involved preliminary denaturation at $95^{\circ} \mathrm{C}$ for $1 \mathrm{~min}$, followed by 45 cycles of $95^{\circ} \mathrm{C}$ for $30 \mathrm{sec}$. The annealing temperature was reduced to $57^{\circ} \mathrm{C}$ for $30 \mathrm{sec}$ and $72^{\circ} \mathrm{C}$ for $10 \mathrm{~min}$. The reaction volume was a total of $20 \mu \mathrm{l}$ containing $40 \mathrm{ng}$ genomic cDNA, $100 \mu \mathrm{M}$ dNTPs, $200 \mu \mathrm{M}$ primers and Taq DNA polymerase and SYBR-Green (each, $2.5 \mathrm{U}$; Thermo Fisher Scientific, Inc.). The forward and reverse primers were synthesized by Invitrogen (Thermo Fisher Scientific, Inc.) and are listed in Table I. Relative mRNA expression levels were calculated using the $2^{-\Delta \Delta \mathrm{Cq}}$ method (17).

Western blotting. On day 21 , TGF- $\beta$, TGF- $\beta$ IR and/or resveratrol-treated neurons were lysed using radioimmunoprecipitation buffer containing protease inhibitors (Thermo Fisher Scientific, Inc.) at $4^{\circ} \mathrm{C}$ for $10 \mathrm{~min}$. Protein concentration was measured using a bicinchoninic acid assay kit (Thermo Fisher Scientific, Inc.). Total protein (30 $\mu \mathrm{g} /$ lane) was separated via SDS-PAGE on $15 \%$ gels. The separated proteins were then transferred to polyvinylidene difluoride membranes (EMD Millipore, Billerica, MD, USA) and incubated in blocking buffer (5\% bovine serum albumin; Sigma-Aldrich; Merck $\mathrm{KGaA}$ ) for $2 \mathrm{~h}$ at $37^{\circ} \mathrm{C}$. Membranes were incubated with the primary antibodies against TGF- $\beta$ (1:1,200; cat. no. ab31013), ERK (1:1,200; cat. no. ab17942), phosphorylated (p-) ERK (1:1,200; cat. no. ab184699), B cell lymphoma-2 (Bcl-2; 1:1,000; cat. no. ab692), B cell lymphoma-extra large (Bcl-xl; 1:1,200; cat. no. ab32370), Bcl-2-associated death promoter (Bad; 1:1,200; cat. no. ab32455), Bcl-2-assocaited X protein (Bax; 1:1,200; cat. no. ab32503), ionized calcium binding adaptor molecule 1 (Iba-1; 1:1,200; cat. no. ab108539), myeloperoxidase (MPO; 1:1,200; cat. no. ab208670) and $\beta$-actin (1:2,000; cat. no. ab8226; all Abcam, Cambridge, UK) for $12 \mathrm{~h}$ at $4^{\circ} \mathrm{C}$. Following incubation, membranes were washed in triplicate with PBS and incubated with horseradish peroxidase (HRP)-conjugated goat anti-rabbit immunoglobulin $\mathrm{G}$ (1:2,000; cat. no. PV-6001; OriGene Technologies, Inc., Rockville, MD, USA) for $1 \mathrm{~h}$ at $37^{\circ} \mathrm{C}$. The membranes were washed three times with PBS. Protein bands were visualized using an enhanced chemiluminescence substrate ECL Select $^{\mathrm{TM}}$ (Roche Applied Science, Penzberg, Germany) and Kodak exposure films (Kodak, Rochester, NY, USA). Protein 
Table I. Primers used in the current study.

\begin{tabular}{ll}
\hline Gene & \multicolumn{1}{c}{ Primer sequence (5'-3') } \\
\hline Bcl-2 & F: 5'-CACAAGAGGCCAAGGCTACC-3' \\
& R: 5'-CAGGAAAGCAGGAAGTCTCAA-3' \\
Bcl-xl & F: 5'-CCAAAATCCCTGCTCTTCATG-3' \\
& R: 5'-GCATTCTTGGCATCGTTATTCA-3' \\
Bax & F: 5'-ATTGAGAAACGATTTGCCTAC-3' \\
& R: 5'-GGAAATGGCTTATTCTCCTTTGCTT-3' \\
Bad & F: 5'-GGAAACCCGGTGGGGCCAC-3' \\
& R: 5'-ACCAGTAGCGGGTGGTC-3' \\
Iba-1 & F: 5'-CACCATTAGCTGGGCGTCT-3' \\
& R: 5'-GATGCGGAAGTAGCAAAAGC-3' \\
MPO & F: 5'-ATTCTCCACACCCTGTTTCG-3' \\
& R: 5'-ATGCAGCAGTGTGTCATTCC-3' \\
TGF- $\beta$ & F: 5'-GGCCAGATCCTGTCCAAGC-3' \\
& R: 5'-GTGGGTTTCCACCATTAGCAC-3' \\
ERK & F: 5'-ACCTAGCCGTGGAGCTTGG-3' \\
& R: 5'-GCCCTTGGTTGTTTACCTGG-3' \\
F-actin & F: 5'-AAGGACCTGTATGCCAACACA-3' \\
& R: 5'-ATCCACACAGAATACTTGCGTT-3' \\
\hline
\end{tabular}

Bcl-2, B cell lymphoma-2; Bcl-xl, B cell lymphoma-extra large; Bax, Bcl-2-assocaited X protein; Bad, Bcl-2-associated death promoter; Iba-1, ionized calcium binding adaptor molecule 1; MPO, myeloperoxidase; TGF- $\beta$; transforming growth factor- $\beta$; ERK, extracellular signal-regulated kinase.

expression was quantified using Quantity-One software (version 1.2; Bio-Rad Laboratories, Inc.).

Immunocytochemistry. Neuronal cells $\left(1 \times 10^{6}\right)$ were fixed in $10 \%$ formaldehyde for $30 \mathrm{~min}$ at $37^{\circ} \mathrm{C}$. Cells were permeabilized with $0.5 \%$ Triton $\mathrm{X}-100$ in $\mathrm{PBS}$ for $5 \mathrm{~min}$ at $25^{\circ} \mathrm{C}$. Endogenous peroxidase activity was blocked using $3 \%$ hydrogen peroxide for $10 \mathrm{~min}$ at room temperature as previously described (18). Subsequently, cells were incubated with specific primary antibodies against Iba-1 (1:1,200; cat. no. ab108539; Abcam) or MPO (1:1,200; cat. no. ab208670; Abcam) for $12 \mathrm{~h}$ at $4^{\circ} \mathrm{C}$. Cells were washed with PBS and subsequently incubated with anti-rabbit HRP-conjugated secondary antibody $(1: 2,000$; cat. no. ab191866; Abcam) for $12 \mathrm{~h}$ at $4^{\circ} \mathrm{C}$. Cells were subsequently washed with PBS three times and incubated with $5 \%$ DAPI (Sigma-Aldrich; Merck KGaA) for $15 \mathrm{~min}$ at $37^{\circ} \mathrm{C}$. Cells were observed under a fluorescent microscope (magnification, x40; Olympus Corporation, Tokyo, Japan). The percentage of positively stained cells was analyzed using Quantity One software (version 4.62; Bio-Rad Laboratories, Inc.).

Terminal deoxynucleotidyl transferase-mediated dUTP nick end labeling (TUNEL) assay. Cells obtained from the rat model of cerebral hemorrhage were analyzed using a TUNEL assay (DeadEnd ${ }^{\mathrm{TM}}$ Colorimetric Tunel System; Promega Corporation, Madison, WI, USA) according to the manufacturer's protocol. Cells were then incubated with $5 \%$ DAPI (Sigma-Aldrich; Merck KGaA) for $15 \mathrm{~min}$ at $37^{\circ} \mathrm{C}$ and washed with PBS three times and mounted onto slides using Aqueous Mounting Medium (Sigma-Aldrich; Merck KGaA). TUNEL-positive cells were observed in three randomly-selected fields under a ZEISS LSM 510 confocal microscope (Zeiss GmbH, Jena, Germany) at $488 \mathrm{~nm}$.

Statistical analysis. Data are presented as the mean \pm standard deviation of triplicate data. All data were analyzed using SPSS 19.0 software (SPSS, IBM Corp., Armonk, NY, USA). Significant differences between two groups were analyzed using a two-tail unpaired Student's t-test. Multiple group differences were analyzed using one-way analysis of variance followed by a Tukey's HSD test. $\mathrm{P}<0.05$ was considered to indicate a statistically significant difference.

\section{Results}

Resveratrol decreases cerebral infarct volume and improves cognitive competence in the rat model of cerebral hemorrhage. The therapeutic effects of resveratrol were analyzed in a rat model of cerebral hemorrhage. The viability assay demonstrated that treatment with resveratrol significantly increased neuronal cell viability in hippocampus tissue compared with the PBS group (Fig. 1A). It was also revealed that resveratrol significantly decreased cerebral infarct volume in the hippocampi of rats with cerebral hemorrhage compared with the PBS group (Fig. 1B). Furthermore, treatment with resveratrol significantly increased the escape latency of rats compared with the PBS group (Fig. 1C). It was also demonstrated that the body weight of resveratrol-treated rats increased significantly compared with the PBS group (Fig. 1D). These results suggest that resveratrol may be an efficient drug for the treatment of cerebral hemorrhage.

Resveratrol improves $C W C$ and $B B B$ disruption in the rat model of cerebral hemorrhage. The results demonstrated that treatment with resveratrol significantly decreased and improved CWC and BBB disruption, respectively, when compared with the PBS group (Fig. 2A-B). Furthermore, treatment with resveratrol significantly increased microglial activation and neutrophil infiltration as determined by Iba-1and MPO-positive cells, respectively (Fig. 2C-D). These results indicate that resveratrol was beneficial for the inhibition of the inflammatory response in the brain of mice with cerebral hemorrhage.

Treatment with resveratrol decreases neuronal cell apoptosis and inflammation. The apoptosis of neurons was assessed following treatment with resveratrol in the TUNEL assay. The results indicated that resveratrol significantly decreased neuronal apoptosis in rats with cerebral hemorrhage (Fig. 3A). Anti-apoptotic gene and protein expression ( $\mathrm{Bcl}-2$ and $\mathrm{Bcl}-\mathrm{xl})$ was significantly upregulated, and pro-apoptotic gene and protein levels (Bad and Bax) were significantly downregulated by treatment with resveratrol, compared with the control (Fig. 3B-C). It was also revealed that treatment with resveratrol significantly increased the expression levels of anti-inflammatory factors Iba-1 and MPO in neurons (Fig. 3D-E). These results indicate that resveratrol decreased neuronal cell apoptosis and inflammation in rats with cerebral hemorrhage. 

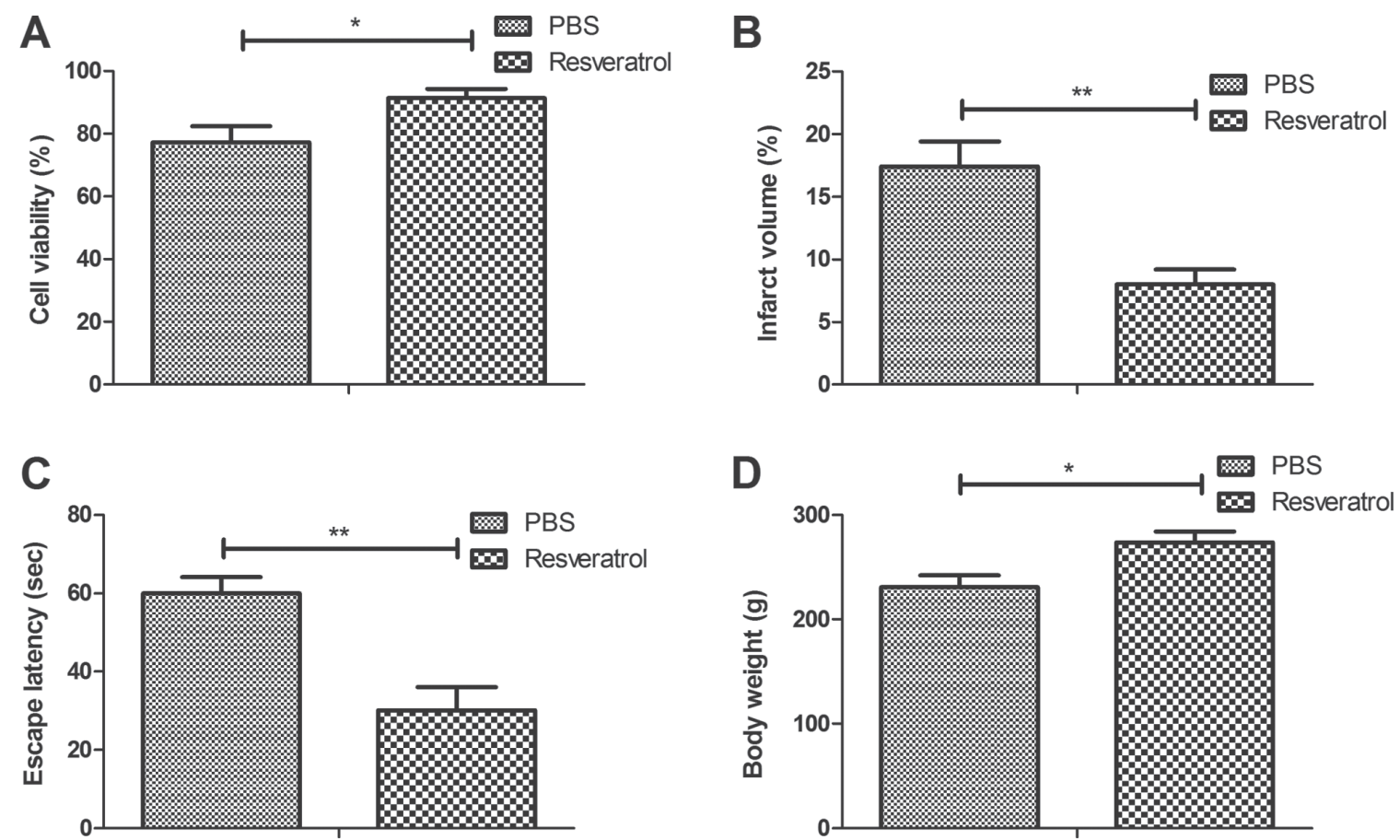

Figure 1. Resveratrol decreases cerebral infarct volume and increases cognitive competence in rats with cerebral hemorrhage. Effects of treatment with resveratrol on (A) the viability of hippocampal neurons, (B) infarct volume, (C) escape latency and (D) body weight of rats with cerebral hemorrhage. ${ }^{*} \mathrm{P}<0.05$ and ${ }^{* *} \mathrm{P}<0.01$.

A
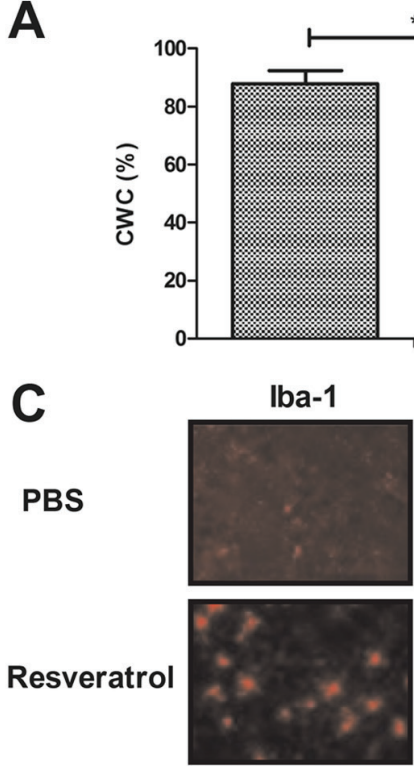

D

PBS

Resveratrol

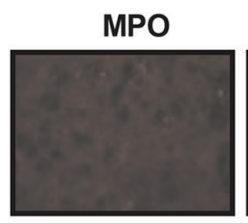

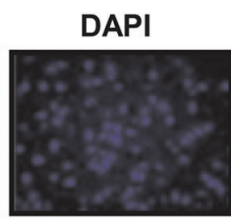

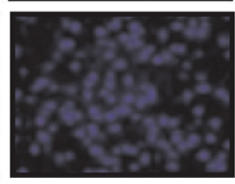

PBS

$\infty$ Resveratrol

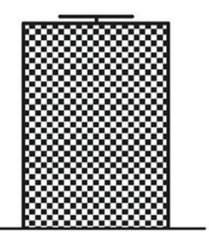

DAPI
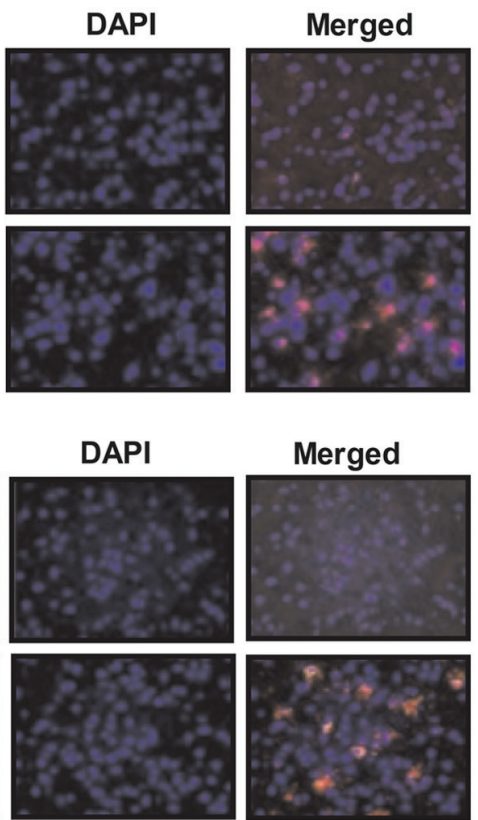

B
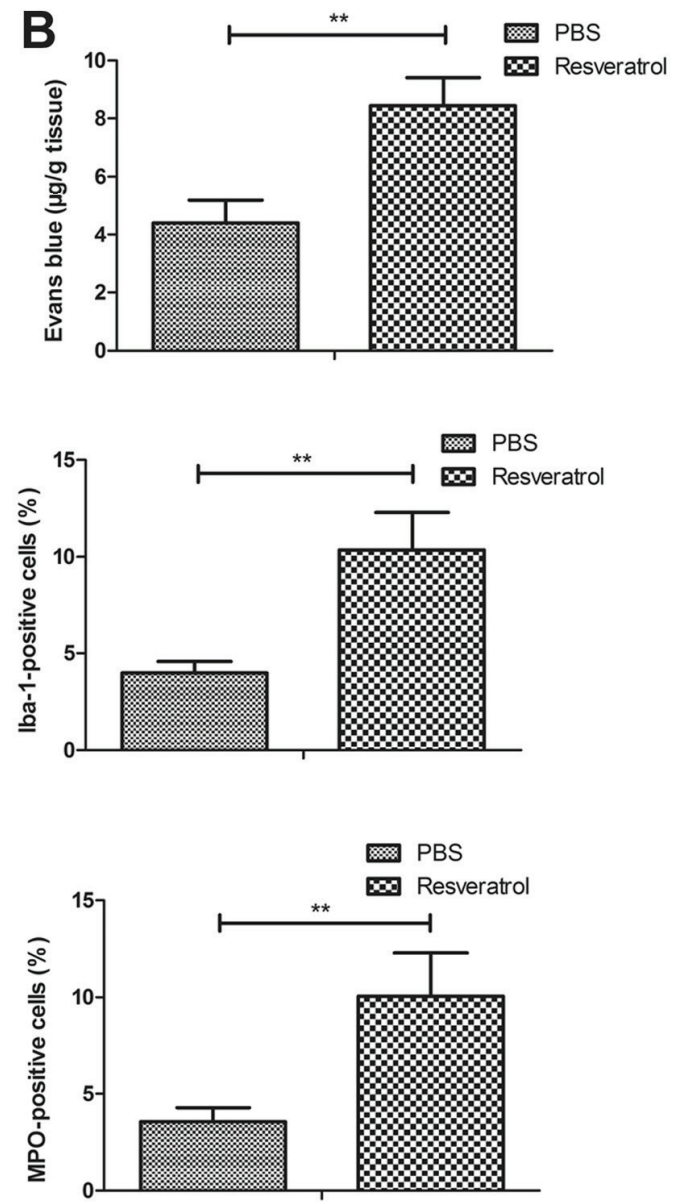

Figure 2. Resveratrol decreases CWC and increases BBB disruption in rats with cerebral hemorrhage. Effect of treatment with resveratrol on (A) CWC and (B) BBB in experimental rats was determined by Evan's blue staining. Effects of treatment with resveratrol on (C) microglial activation and (D) neutrophil infiltration as determined by Iba-1 and MPO-positive cells, respectively (magnification, $\mathrm{x} 40$ ). ${ }^{*} \mathrm{P}<0.05$ and ${ }^{* *} \mathrm{P}<0.01$. CWC, cerebral water content; BBB, blood brain barrier; Iba-1, ionized calcium binding adaptor molecule 1; MPO, myeloperoxidase. 
A

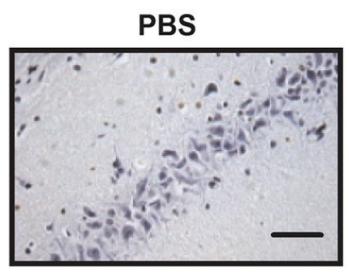

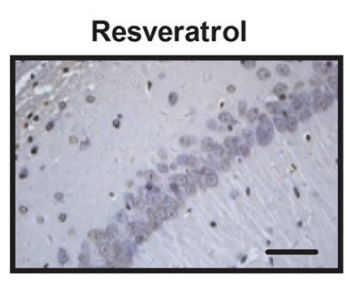
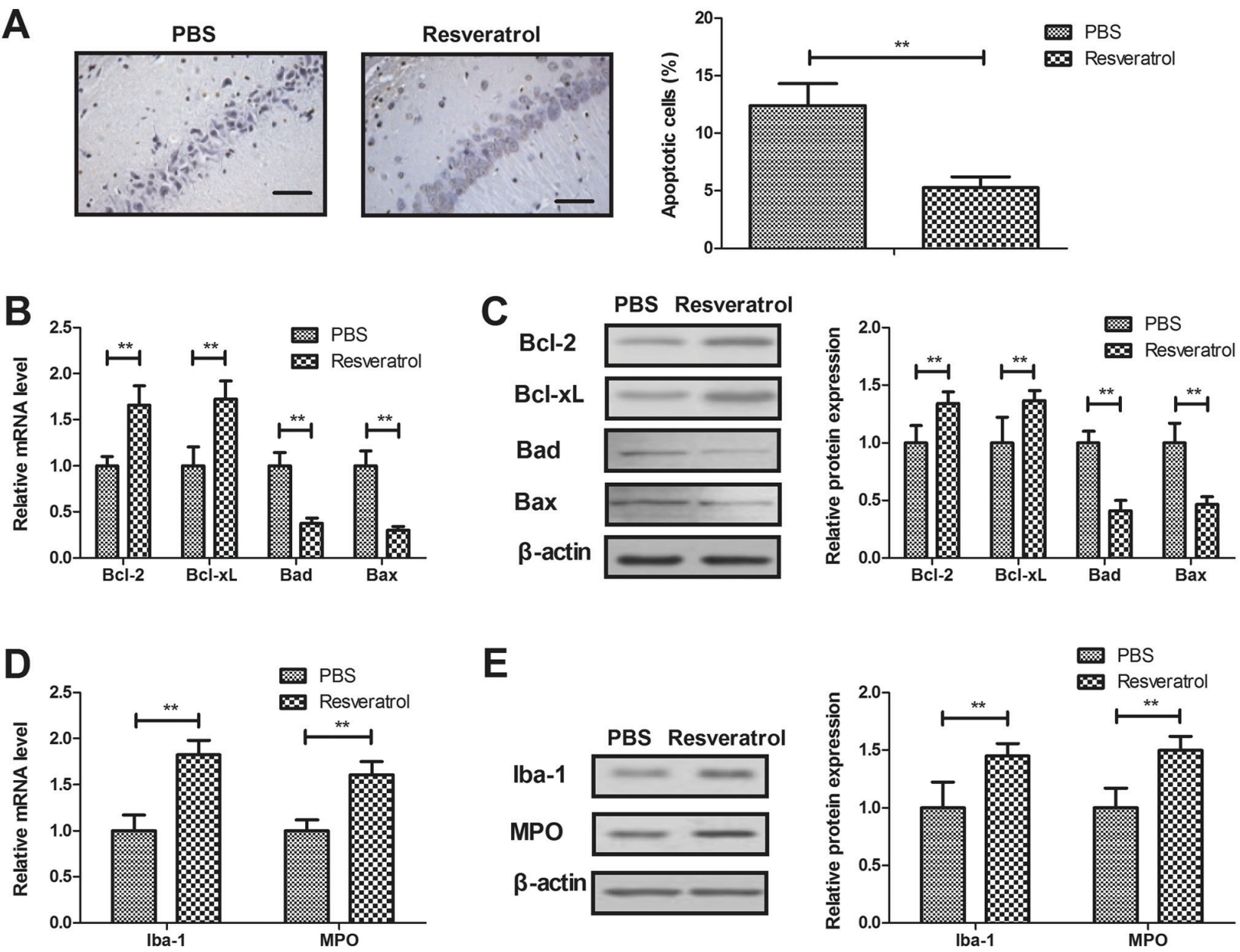

Figure 3. Treatment with resveratrol decreases neuronal cell apoptosis and inflammation. (A) Resveratrol inhibits the apoptosis of neurons isolated from rats with cerebral hemorrhage (magnification, 40x). Effects of resveratrol on anti-apoptotic Bcl-2 and Bcl-xl and pro-apoptotic Bad and Bax (B) mRNA and (C) protein expression in neurons isolated from experimental rats. Effects of treatment with resveratrol on certain anti-inflammatory factors, including Iba-1 and MPO (D) mRNA and (E) protein expression in neurons obtained from rats with cerebral hemorrhage. ${ }^{* *} \mathrm{P}<0.01$. Bcl-2, B cell lymphoma-2; Bcl-xl, B cell lymphoma-extra large; Bad, Bcl-2-associated death promoter; Bax, Bcl-2-assocaited X protein; Iba-1, ionized calcium binding adaptor molecule 1; MPO, myeloperoxidase.

Resveratrol regulates neuronal cell apoptosis by regulating the TGF- $\beta$-mediated ERK signaling pathway. The current study analyzed the potential resveratrol-mediated mechanism of apoptosis and inflammation in neuron cells. It was demonstrated that resveratrol significantly downregulated TGF- $\beta$ and ERK gene and protein expression in neurons isolated from rats with cerebral hemorrhage (Fig. 4A-B). Treatment with TGF- $\beta$ IR downregulated TGF- $\beta$ and ERK gene and protein expression levels in neurons (Fig. 4C-D). Resveratrol-TGF- $\beta$ treatment increased the protein expression levels of ERK/pERK compared with TGF- $\beta$ treatment alone (Fig. 4E). In addition, resveratrol-TGF- $\beta$ treatment significantly decreased the number of TUNEL positive cells compared with TGF- $\beta$ treatment (Fig. 4F) The results indicated that treatment with TGF- $\beta$ reversed the decrease in neuronal cell apoptosis induced by resveratrol (Fig. 4F). These results suggest that resveratrol decreased apoptosis by downregulating the TGF- $\beta$-mediated ERK signaling pathway.

\section{Discussion}

Neuronal apoptosis serves a crucial role in the progression of brain injury (19). A previous study has demonstrated that resveratrol prevents neuronal apoptosis in an early brain injury model through the phosphoinositide 3-kinase/protein kinase B (PI3K/Akt) signaling pathway (20). The current study assessed the therapeutic effects of resveratrol in a rat model of cerebral hemorrhage induced by reperfusion injury and further analyzed the potential mechanism of resveratrol in neuronal cells. The results demonstrated that resveratrol decreased apoptosis and inflammation in rats of the hippocampal reperfusion injury model. The results also indicated that resveratrol inhibited hippocampal cell apoptosis and inflammation by downregulating the of TGF- $\beta$-ERK signaling pathway.

Evidence has indicated that the inhibition of neural apoptosis attenuated early brain injury induced by subarachnoid hemorrhage (21). A previous study has demonstrated that resveratrol inhibited b-amyloid-induced neuronal apoptosis by regulating the acetylation of cellular tumor antigen p53 in PC12 cells (22). It has also been indicated that increasing the activity of the Bak signaling pathway may mediate apoptosis in cultured neural progenitor cells (23). In the current study, treatment with resveratrol inhibited hippocampal cell apoptosis by decreasing the expression of Bad and Bax in neurons. Additionally, treatment with resveratrol upregulated 
A

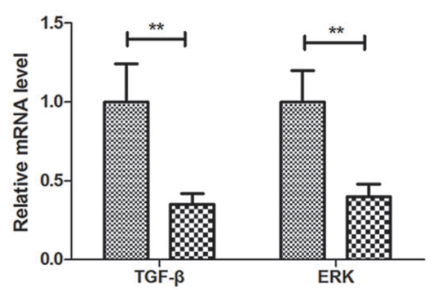

C

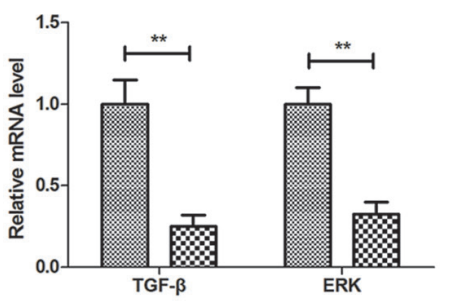

E

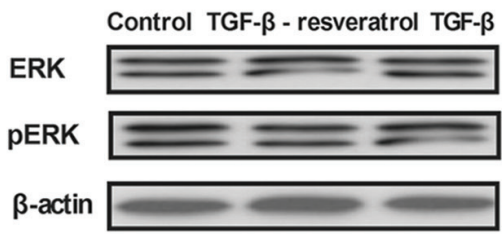

$\mathbf{F}$

Control

TGF- $\beta$ - resveratrol
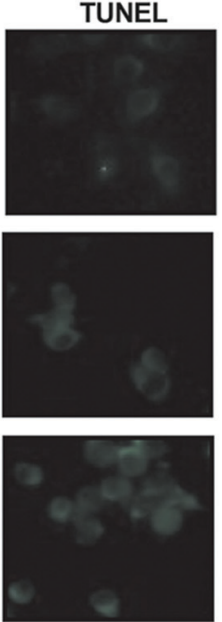

TGF- $\beta$
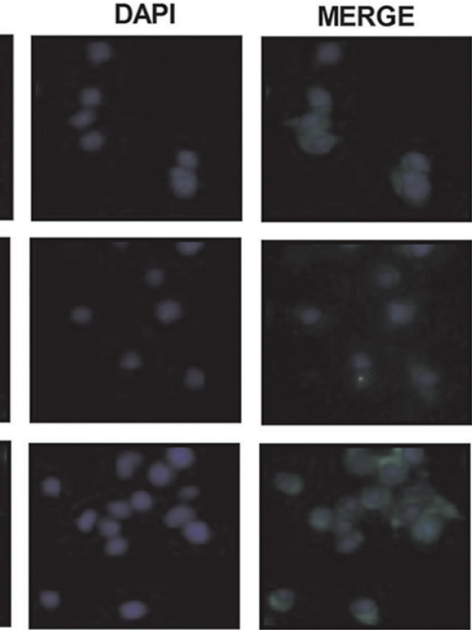

B

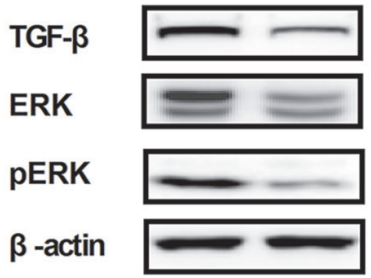

D

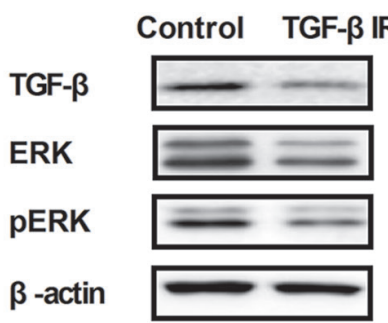

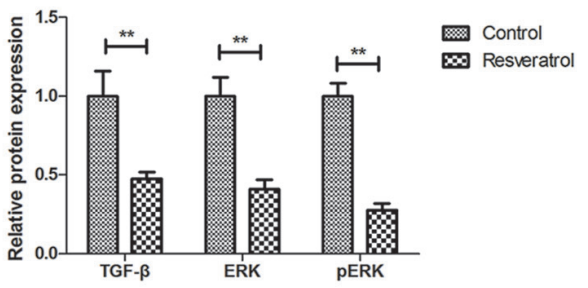

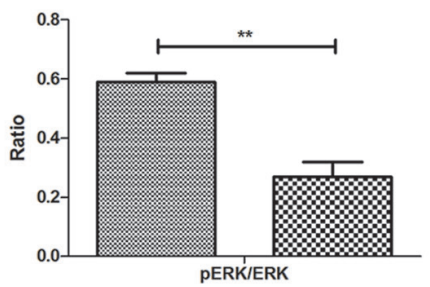

Control

$\infty$ Resveratrol
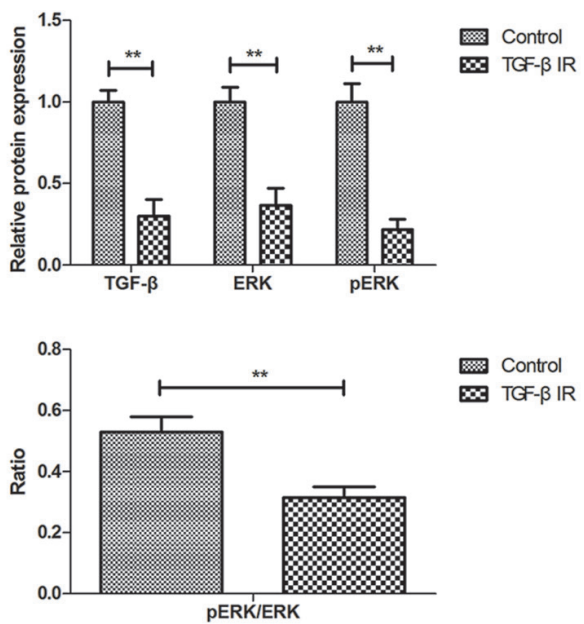

Control $\times \mathbb{T G F}-\beta I R$

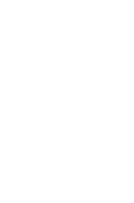

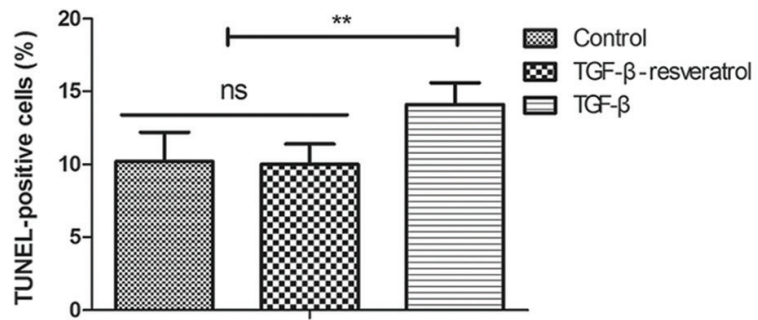

Figure 4. Resveratrol regulates neuronal cell apoptosis by regulating the TGF- $\beta$-mediated ERK signaling pathway. Effects of resveratrol on TGF- $\beta$ and ERK (A) mRNA and (B) protein expression in neurons isolated from rats with cerebral hemorrhage. Effect of TGF- $\beta$ inhibitor on the TGF- $\beta$ and ERK (C) mRNA and (D) protein levels. (E) Effects of treatment with TGF- $\beta$ on resveratrol-decreased ERK and pERK expression in neurons. (F) Effects of treatment with TGF- $\beta$ on neuronal apoptosis. " $\mathrm{P}<0.01$. TGF- $\beta$, transforming growth factor- $\beta$; ERK, extracellular signal-regulated kinase; TGF- $\beta$ IR, TGF- $\beta$ inhibitor; ns, not significant. 
anti-apoptotic Bcl-2 and Bcl-xl protein levels, which may be the reason for the resveratrol-induced decrease of apoptosis in neurons.

Previous studies have revealed that TGF- $\beta$ promotes recovery following intracerebral hemorrhage, indicating that TGF- $\beta$ may be a therapeutic target for the treatment of acute brain injury (24). The current study demonstrated that treatment with resveratrol decreased the expression of TGF- $\beta$, which further led to the decrease of neuronal cells apoptosis. A further study has revealed that ERK serves a protective role in the apoptotic mechanisms involved in sympathetic neuron survival (25). The results of the present study demonstrated that treatment with resveratrol inhibits hippocampal cell apoptosis by downregulating the TGF- $\beta$-mediated ERK signaling pathway. Li et al (18) revealed that nerve growth factor attenuates high glucose-induced endoplasmic reticulum stress, which prevents Schwann cells from undergoing apoptosis by activating the PI3K/Akt/glycogen synthase kinase-3 $\beta$ and ERK1/2 signaling pathways. Notably, the present study demonstrated that treatment with resveratrol decreased cerebral infarct volume and CWC. It also improved cognitive competence and BBB disruption in the rat model of cerebral hemorrhage. However, the current study did not assess the effects of an ERK inhibitor on resveratrol-treated brain injury. Further study is therefore required to analyze the role of an ERK inhibitor on the resveratrol-induced decrease of hippocampal cell apoptosis in cerebral hemorrhage.

In conclusion, the current study assessed the therapeutic effects of resveratrol in hippocampal cells of rats with cerebral hemorrhage. The results revealed that treatment with resveratrol inhibited neuronal cell apoptosis by downregulating the TGF- $\beta$-mediated ERK signaling pathway in rats with cerebral hemorrhage. These results indicate that resveratrol may be a potential drug for the treatment of cerebral hemorrhage induced by ischemic reperfusion.

\section{Acknowledgements}

Not applicable.

\section{Funding}

No funding was received.

\section{Availability of data and materials}

The datasets used and/or generated during the present study are available from the corresponding author on reasonable request.

\section{Authors' contributions}

RZ performed the experiments. KZ, HS and PZ analyzed all of the data generated in this study. NZ designed the study and prepared this manuscript.

\section{Ethics approval and consent to participate}

The present study was approved by the Ethics Committee of Tianjin Baodi Hospital (Tianjin, China).

\section{Patient consent for publication}

Not applicable.

\section{Competing interests}

All authors declare that they have no competing interests.

\section{References}

1. Al-Mufti F, Amuluru K, Changa A, Lander M, Patel N, Wajswol E, Al-Marsoummi S, Alzubaidi B, Singh IP, Nuoman R and Gandhi C: Traumatic brain injury and intracranial hemorrhage-induced cerebral vasospasm: A systematic review. Neurosurgical focus 43: E14, 2017.

2. Shoamanesh A, Kwok CS, Lim PA and Benavente OR: Postthrombolysis intracranial hemorrhage risk of cerebral microbleeds in acute stroke patients: A systematic review and meta-analysis. Int J Stroke 8: 348-356, 2013.

3. Bugeme M and Mukuku O: Neuropsychiatric manifestations revealing cerebral subarachnoid hemorrhage caused by electrification accident about a case and review of literature. Pan AfrMed J 18: 201, 2014 (In French).

4. Wang X, Gao XD, Gao B, Jia LL, Yang MF, Zhang YB and Sun BL: Cerebral lymphatic blockage aggravates apoptosis of cortical neurons after subarachnoid hemorrhage in rats in vivo. Sheng Li Xue Bao 62: 109-114, 2010 (In Chinese).

5. Jeong SI, Shin JA, Cho S, Kim HW, Lee JY, Kang JL and Park EM: Resveratrol attenuates peripheral and brain inflammation and reduces ischemic brain injury in aged female mice. Neurobiol Aging 44: 74-84, 2016.

6. Li H, Yan Z, Zhu J, Yang J and He J: Neuroprotective effects of resveratrol on ischemic injury mediated by improving brain energy metabolism and alleviating oxidative stress in rats. Neuropharmacology 60: 252-258, 2011.

7. Zou P, Liu X, Li G and Wang Y: Resveratrol pretreatment attenuates traumatic brain injury in rats by suppressing NLRP3 inflammasome activation via SIRT1. Mol Med Rep 17: 3212-3217, 2018.

8. Karalis F, Soubasi V, Georgiou T, Nakas CT, Simeonidou C, Guiba-Tziampiri O and Spandou E: Resveratrol ameliorates hypoxia/ischemia-induced behavioral deficits and brain injury in the neonatal rat brain. Brain Res 1425: 98-110, 2011.

9. Logan TT, Villapol S and Symes AJ: TGF- $\beta$ superfamily gene expression and induction of the Runx1 transcription factor in adult neurogenic regions after brain injury. PLoS One 8: e59250, 2013.

10. Sugawara T, Jadhav V, Ayer R and Zhang J: Simvastatin attenuates cerebral vasospasm and improves outcomes by upregulation of PI3K/Akt pathway in a rat model of subarachnoid hemorrhage. Acta Neurochir Suppl 102: 391-394, 2008.

11. Aguilar-Alonso P, Vera-Lopez O, Brambila-Colombres E, Segura-Badilla O, Avalos-López R, Lazcano-Hernández M and Navarro-Cruz AR: Evaluation of oxidative stress in cardiomyocytes during the aging process in rats treated with resveratrol. Oxid Med Cell Longev 2018: 1390483, 2018.

12. Gamberini MT, Rodrigues DS, Rodrigues D and Pontes VB: Effects of the aqueous extract of Pimpinella anisum L. seeds on exploratory activity and emotional behavior in rats using the open field and elevated plus maze tests. J Ethnopharmacol 168: 45-49, 2015.

13. Vatter H, Weidauer S, Konczalla J, Dettmann E, Zimmermann M, Raabe A, Preibisch C, Zanella FE and Seifert V: Time course in the development of cerebral vasospasm after experimental subarachnoid hemorrhage: Clinical and neuroradiological assessment of the rat double hemorrhage model. Neurosurgery 58: 1190-1197; discussion 1190-1197, 2006.

14. Hijioka M, Matsushita H, Hisatsune A, Isohama Y and Katsuki H: Therapeutic effect of nicotine in a mouse model of intracerebral hemorrhage. J Pharmacol Exp Ther 338: 741-749, 2011.

15. Hong H, Kim CJ, Kim JD and Seo JH: $\beta$-glucan reduces exercise-induced stress through downregulation of c-Fos and c-Jun expression in the brains of exhausted rats. Mol Med Rep 9: 1660-1666, 2014.

16. Chen H, Guan B, Chen X, Chen X, Li C, Qiu J, Yang D, Liu KJ, Qi S and Shen J: Baicalin attenuates blood-brain barrier disruption and hemorrhagic transformation and improves neurological outcome in ischemic stroke rats with delayed t-PA treatment: Involvement of ONOO(-)-MMP-9 pathway. Transl Stroke Res 9: 515-529, 2017. 
17. Livak KJ and Schmittgen TD: Analysis of relative gene expression data using real-time quantitative PCR and the 2(-Delta Delta C(T)) method. Methods 25: 402-408, 2001.

18. Li R, Wu Y, Zou S, Wang X, Li Y, Xu K, Gong F, Liu Y, Wang J, Liao Y, et al: NGF attenuates high glucose-induced ER stress, preventing schwann cell apoptosis by activating the PI3K/Akt/GSK3 $\beta$ and ERK1/2 pathways. Neurochem Res 42: 3005-3018, 2017.

19. Hausmann R, Biermann T, Wiest I, Tubel J and Betz P: Neuronal apoptosis following human brain injury. Int J Legal Med 118: 32-36, 2004.

20. Zhou XM, Zhou ML, Zhang XS, Zhuang Z, Li T, Shi JX and Zhang X: Resveratrol prevents neuronal apoptosis in an early brain injury model. J Surg Res 189: 159-165, 2014

21. Song J, Cho KJ, Cheon SY, Kim SH, Park KA, Lee WT and Lee JE: Apoptosis signal-regulating kinase 1 (ASK1) is linked to neural stem cell differentiation after ischemic brain injury. Exp Mol Med 45: e69, 2013.

22. Ai Z, Li C, Li L and He G: Resveratrol inhibits beta-amyloidinduced neuronal apoptosis via regulation of p53 acetylation in PC12 cells. Mol Med Rep 11: 2429-2434, 2015.
23. Chen X, Liu J, Feng WK, Wu X and Chen SY: MiR-125b protects against ethanol-induced apoptosis in neural crest cells and mouse embryos by targeting Bak 1 and PUMA. Exp Neurol 271: 104-111, 2015.

24. Taylor RA, Chang CF, Goods BA, Hammond MD, Mac Grory B Ai Y, Steinschneider AF, Renfroe SC, Askenase MH, McCullough LD, et al: TGF- $\beta 1$ modulates microglial phenotype and promotes recovery after intracerebral hemorrhage. J Clin Invest 127: 280-292, 2017.

25. Anderson CN and Tolkovsky AM: A role for MAPK/ERK in sympathetic neuron survival: Protection against a p53-dependent, JNK-independent induction of apoptosis by cytosine arabinoside. J Neurosci 19: 664-673, 1999.

This work is licensed under a Creative Commons Attribution-NonCommercial-NoDerivatives 4.0 International (CC BY-NC-ND 4.0) License. 